\title{
Identity adaptation is mediated and moderated by visualisation ability
}

\author{
Peter J Hills \\ Department of Psychology, Anglia Ruskin University, Broad Street, Cambridge CB1 1PT, UK; \\ e-mail: peter.hills@anglia.ac.uk

\section{Rachael L Elward, Michael B Lewis} \\ School of Psychology, Cardiff University, Tower Building, Park Place, Cardiff CF10 3AT, Wales, UK \\ Received 4 June 2007, in revised form 3 April 2008
}

\begin{abstract}
Depending on the previous research one reads, face aftereffects may or may not partially transfer across viewpoints. Two experiments are reported that explore this face-identity aftereffect by varying the adaptor type. Adaptation occurs when different views of the identity are used and even when the person's name is the adaptor. Brief exposure or nationality did not produce adaptation. In a third experiment, the role of visualisation was explored. Participants with higher visualisation scores showed greater adaptation to names than those with lower scores. These findings suggest non-facial identity cues can lead to a facial adaptation effect and that visualisation may be the mechanism behind this.
\end{abstract}

\section{Introduction}

Adaptation (the reduction of neuronal responsiveness due to prolonged exposure of a particular stimulus feature-Sekuler and Blake 2001) is a tool frequently used to study visual perception and has recently been applied to higher-level representations of objects and faces (eg Hurlbert 2001). Adaptation to a distorted face causes an average face to appear distorted in the opposite direction to that distortion (Webster and MacLin 1999). For example, a compressed face used as the adaptor will cause a postadaptation average face to appear expanded (eg Rhodes et al 2003). This effect is commonly known as the face-distortion aftereffect (FDAE; eg Zhao and Chubb 2001). Similar to FDAEs are face-identity aftereffects (FIAEs), whereby the perceived identity of a face is 'opposite' after adaptation to a particular identity.

Leopold et al (2001) conducted a pioneering study into the FIAE. In their study, 200 faces were morphed together to produce a prototype face. Because of the morphing process, each face identity could then be measured in terms of distances from the prototype face in a face-space (cf Valentine 1991). Some of the faces were selected to be used as targets, and a series of faces were created, ranging from the prototype face to each target face identity, each differing in identity 'strength'. Identification thresholds (the required identity strength to perceive the face identity) were taken before and after adaptation to an anti-face identity (opposite from the face identity in terms of Euclidean geometry). After adaptation to the anti-face the identification threshold was lowered by $12.5 \%$, suggesting it was easier to perceive the identity following adaptation to the anti-face identity.

The magnitude of FIAE is typically measured in terms of difference in identification thresholds before and after adaptation and is dependent on the presentation duration of both the adaptor and the test stimuli (Leopold et al 2005). Stronger FIAEs were observed when the adaptors were presented for longer durations (16000 ms) than for shorter durations (1000 ms). Moreover, FIAEs were significantly stronger when the test stimuli were presented for shorter durations $(100 \mathrm{~ms})$ than for longer durations (1600 ms). Jiang et al (2007) demonstrated that the FIAE transfers across a substantial 
change in viewpoint $\left(30^{\circ}\right.$ rotation), indicating the mechanisms for the FIAE are of a high-level nature (see also Pourtois et al 2005; Ryu and Chaudhuri 2006).

There is some debate, however, whether face aftereffects actually transfer across viewpoints. Benton et al (2006) and Jeffery et al (2006) found that the magnitude of the FDAE was greater for faces tested in the same pose as the adaptor face than for faces tested in a different pose to the adaptor face. Both research teams assert that the FDAE is viewpoint-dependent. However, their conclusion is unwarranted for two reasons. First, the magnitude of the FDAE was significantly greater than zero for faces tested in a different pose from the adaptor face: using a normality rating procedure, Jeffery et al (2006) reported that judgments to a post-adaptation face were altered by nine points on the rating scale for faces tested in the same view as the adaptor, whereas the judgments were altered by four points for faces tested in a different view (from three-quarter left to frontal or three-quarter right). That is, $44 \%$ of the FDAE is viewpoint-independent and this is the same whether the face is rotated by $45^{\circ}$ or $90^{\circ}$. Second, there were substantial individual differences in Benton et al's (2006) data, whereby one participant showed the same magnitude of the FDAE for faces tested in the same pose as and different pose from the adaptor face, and one participant showed no adaptation to faces tested in a different pose from the adaptor face. Such individual variability was not discussed by these authors.

In contrast to the results of Benton et al (2006), Carbon and Leder (2005) have shown that the FDAE in a familiar face (Princess Diana) does reliably transfer to novel viewpoints. In addition to using familiar rather than unfamiliar faces, Carbon and Leder used more participants than Benton et al and all their participants were naive to the purpose of their experiment. One purpose of the present work is to establish, by using two different methodologies that can thus increase confidence in any effect, whether face aftereffects will transfer across viewpoints.

Within face recognition, it is well established that non-visual information can affect face processing. Priming is whereby prior presentation of a stimulus speeds up the processing of the same or a related stimulus and has been used to explore the multimodel architecture of the face-recognition system. For example, Ellis et al (1987) demonstrated that familiarity judgments made to a face were quicker if that face had been primed previously. The magnitude of this priming effect was dependent on the perceptual similarity between the prime and the target: response times were faster to the target face when the prime and target face were in the same pose than when they were in different poses. Additionally, Young et al (1994) have shown a cross-modal priming effect, whereby written names sped up familiarity judgments of faces when the prime preceded the face by a short period of time. Johnston and Barry (2006) have shown that cross-modal repetition priming effects are significantly smaller than within-modal priming effects, but are still significant themselves. Given that the recognition of faces can be influenced by the presentation of non-visual information, a second purpose of the present work was to explore any possible influence non-visual information may have on adaptation to a particular face identity

Returning to the FIAE, Moradi et al (2005) suggest that the FIAE requires conscious perception. Moradi et al tested the effect different types of suppression had on the magnitude of the FIAE. Though the FIAE transferred from the adapted retina to the unadapted retina, it disappears when the participants are attending to the eye that is not being adapted. For example, one eye is being adapted to a face identity, while the other eye is presented with a pattern of moving random dots. Participants who attended to the moving pattern often ignored the face and failed to show the FIAE.

Moradi et al (2005) also tested whether imagination can cause the FIAE. Six participants were trained to associate names with the anti-faces of Leopold et al (2001). 
This training lasted 300 trials. They were then asked to imagine one of the faces and were asked how clear their mental image was. Participants reported that their visualisation was vivid and yet demonstrated no FIAE even after prolonged visual imagery. This observation is apparently at odds with the fact that there is activation in the visual cortex during mental imagery (Kosslyn et al 1993, 1994). In addition, the fusiform gyrus [the 'face-specific' area of the inferotemporal cortex-Kanwisher et al 1997] shows activation during actual perception of faces and during mental imagery of faces (Kreiman et al 2000; O'Craven and Kanwisher 2000). Since there are significant individual differences in the ability to mentally visualise (eg Amedi et al 2005; Bywaters et al 2004; Hasnain and Husain 1980; Issac and Marks 1994; McKelvie 1994; Richardson 2000; Richardson and McAndrew 1990) and in the activation of the visual cortex during imagery (cf Cui et al 2007; Ishai et al 2005), it is possible that the small sample (six participants) tested by Moradi et al was affected by a single participant unable to accurately visualise a particular face. Additionally, training participants to associate a name with a 2-D digitised face that has only ever been seen in one pose is unlikely to lead to an accurate face representation (cf Burton et al 2005). Moreover, the assessment of visualisation clarity was not based on previous mental imagery work (eg Marks 1973). Indeed, the failure of Benton et al (2006) to find aftereffects when the test stimuli did not match the adaptor in terms of pose may be related to this individual difference, since people may mentally rotate a threequarter-view face to a frontal view (cf Kersten et al 1996; Troje and Bülthoff 1996). A third purpose of the present paper is to explore the role of visualisation in the FIAE.

Three experimental paradigms are employed here to address the three issues discussed: whether the FIAE can transfer across viewpoints; whether the FIAE can be influenced by non-visual information; and whether the FIAE is affected by participants' visualisation abilities. In experiment 1 we use a two-alternative forced-choice procedure to identify cross-modal adaptations, having pre-exposed participants to the identities to be used. In experiment 2 we use the same procedure but the participants are not pre-exposed to the identities, thus they do not know who the 'opposite' identity is. This methodology is not commonly used to assess face aftereffects but has been used for other tests of visual illusions and related aftereffects (eg Hanley and Zerbolio 1965; cf Spillmann 1994). In experiment 3 we use a psychometric procedure to test whether the individual differences in identity adaptation effects are due to the participants' ability to mentally visualise. In experiment 3 we also test whether parts of identity adaptation are due to visualisation. To do this, pictures of two familiar faces were morphed together, creating a 50\% image. Familiar faces were used, since this requires less training and fewer instructions. Participants were adapted only once to prevent demand characteristics, as after one condition participants will be aware of the experimental hypothesis.

\section{Experiment 1}

In experiment 1 we assess whether viewpoint differences affect the FIAE. The conclusions of Benton et al (2006) and Jeffery et al (2006) indicate that a change in viewpoint between adaptation and test should remove the FIAE. In addition, in experiment 1 we assessed whether names can influence the FIAE. According to the priming literature, it seems plausible that names may influence the FIAE, whereas Moradi et al's (2005) claim that the face needs to be attended to in order that an aftereffect be caused suggests that only the face will cause the FIAE. In experiment 1 we employ a onetrial-per-subject methodology (similar to Hanley and Zerbolio 1965). This deviates from the customary method for assessing the FIAE and is used for two reasons: first to ensure that the face aftereffect is generalisable and reliable under different 
experimental procedures; and second to use similar methodologies to those of priming studies. Priming studies tend to present one particular identity once in the experimental phase. Thus, in the current methodology we present the identity once. If multiple identities were presented, it was felt that, following the first presentation, participants would become aware of the adaptation manipulation and may respond in a manner consistent with the experimenters' expectations. Additionally, evidence from certain visual illusions indicates that repeated presentation and prior knowledge of an effect affects the magnitude of subsequent aftereffects (cf Favreau 1979; Jones and Holding 1975). As such, we employed a one-trial-per-subject methodology for experiments 1 and 2.

\subsection{General method}

2.1.1 Participants. Participants consisted of one hundred staff and students at Cardiff University with normal or corrected vision who participated voluntarily. Twenty participants took part in each condition.

2.1.2 Materials. Two different images of Pierce Brosnan and Harrison Ford were collected. One image of Pierce Brosnan was paired with one image of Harrison Ford, and the second image of Pierce Brosnan was paired with the second image of Harrison Ford on the basis of the image dimensions (pair A was $83 \mathrm{~mm} \times 140 \mathrm{~mm}$; pair B was $100 \mathrm{~mm} \times 100 \mathrm{~mm}$ ), pose (pair A were frontal with slight tilt to the right; pair B were three-quarter facing to the left), and lighting (pair A was lit from the left; pair B was lit from the front). All images had a resolution of 72 pixels inch $^{-1}$. Each pair was morphed together with Smartmorph ${ }^{(i x}$ software, by means of 200 anchor points. A series of morphs was created that ranged from $100 \%$ Harrison Ford to $100 \%$ Pierce Brosnan in increments of $5 \%$. These were pretested (by two additional naive observers) by using a staircase procedure, such that the point that was rated as Harrison Ford on $50 \%$ trials was found. Both observers rated the $55 \%$ Harrison Ford for image pair A, and the $45 \%$ Harrison Ford for image pair B as the point of stimulus equality (PSE). We used these perceptual midpoints as the test stimuli. The $100 \%$ images for each identity and each pair were used as the adaptors. All stimuli were presented on a high-resolution colour monitor connected to an RM PC running Microsoft Windows $\mathrm{XP}^{\circledR}$ with Powerpoint ${ }^{(i x}$ presentation software.

2.1.3 Design. The dependent variable for the experiments was identity response given to the midpoint image. The adaptors were varied between subjects: half the participants were adapted to Harrison Ford, the other half were adapted to Pierce Brosnan; half the participants were tested on image pair A, whereas the other half were tested on image pair B. Each participant saw only one adaptor. A $\chi^{2}$ test revealed that there was no effect of identity (48\% Harrison Ford responses) or image pair. As such, the data were pooled across these variables. In each experiment, chance levels would be indicated by $50 \%$ of the (10 out of 20) participants giving a response as the non-adaptor.

2.1.4 Procedure. Participants were tested individually or in groups of up to three. The procedure consisted of three short phases: the instructions; the presentation of the adaptor; and the test phase. In the instructions, participants were shown a picture of Harrison Ford and informed who he was, followed by a picture of Pierce Brosnan and his name. The faces and the names were on screen for $1 \mathrm{~s}$ each. All participants knew the identities. Participants were then instructed to examine the adaptor for the duration it was on screen (60 s unless otherwise stated). Immediately following the adaptor, the test morph appeared on screen and remained on screen until the participant gave a response. Participants were instructed to write down the initials of the person the morph looked most like, and could take as long as they required. This never took longer than $20 \mathrm{~s}$. Figure 1 shows a demonstration of this task. 


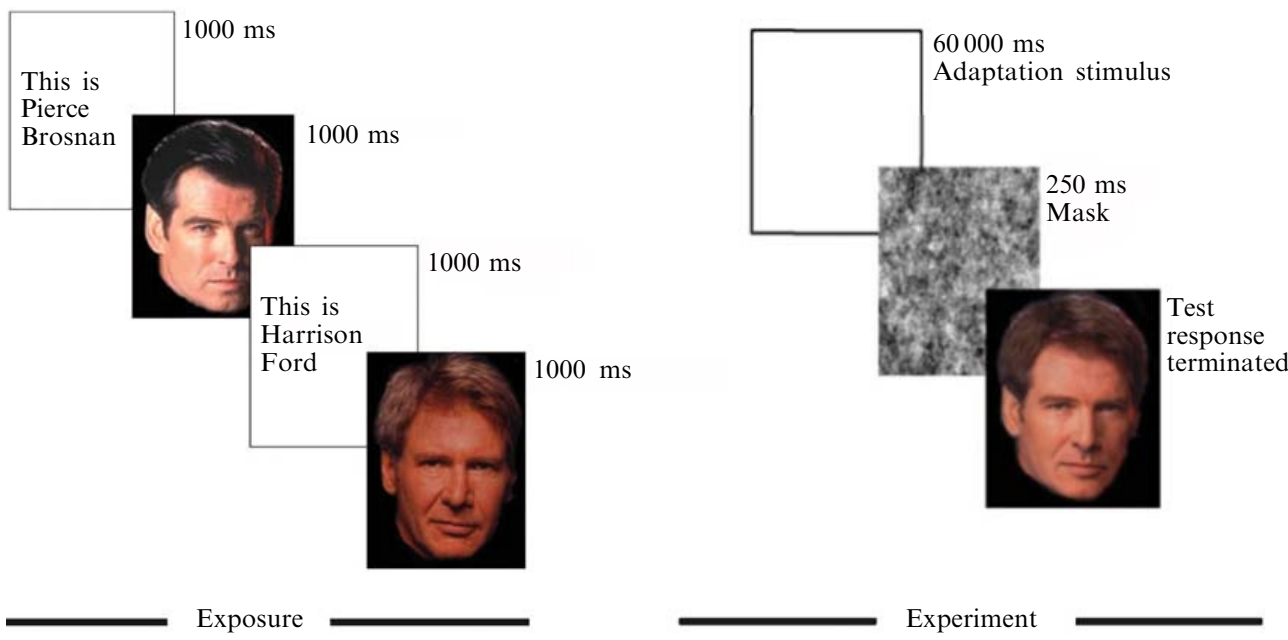

Figure 1. [In colour online, see http://dx.doi.org/10.1068/p5834] Procedure for experiment 1.

\subsection{Condition 1-same image}

This condition acted as a replication of the Leopold et al (2001) study, testing whether FIAEs are reliable for familiar faces.

2.2.1 Method. The method was as described above. The adaptor was the same as that used to construct the morph.

2.2.2 Results. If participants were unaffected by the adaptor, then the midpoint image would be identified as identity at chance levels $(50 \%$; 10 out of 20 participants). This was not the case. $90 \%$ (18 out of 20) of participants gave a response that was not the adaptor identity. This was significantly different to chance and reflects adaptation taking place $\left(\chi_{1}^{2}=7.619, p<0.01\right)$.

\subsection{Condition 2-different image}

In this condition we extended the adaptation procedure by using different images at adaptation and test. This acts as a replication of the Benton et al (2006) and the Jeffery et al (2006) studies.

2.3.1 Method. The general method was applied, but the adaptor was not the same as that used to construct the morph. That is, there was a pose change from the adaptation phase to the test phase.

2.3.2 Results and discussion. Adaptation to a different image to the one used in the morph caused significant adaptation $\left(\chi_{1}^{2}=7.619, p<0.01\right)$, again with $90 \%$ of participants choosing the unadapted morph identity (18 out of 20). This effect is indicative of the viewpoint-independent representation of familiar faces (cf Ryu and Chaudhuri 2006).

\subsection{Condition 3-written name}

Identity is made up from many different aspects, including voices, names, biographical information, and the visually derived aspects. In condition 3 we set out to test whether the FIAEs can be caused by other aspects of identity.

2.4.1 Method. Instead of using an image as the adaptor, we used the written name (presented in the middle of the screen for $60 \mathrm{~s}$ ). All other aspects of the method were as described in the general method. 
2.4.2 Results and discussion. 80\% of responses given were the non-adapted identity when the adaptor was the name (16 out of 20). This was not significantly different from the number of participants adapted when the face was the adaptor. It was significantly different from chance $\left(\chi_{1}^{2}=3.956, p<0.05\right)$.

When the adaptor is a written name, it causes FIAEs of similar magnitude to those caused by images. Responses to a visually presented face are being altered owing to the prolonged exposure of the adaptation identity, irrespective of the modality of presentation. This important effect may well be controversial and demand characteristics may play a role in this study: that is, observers may be responding with a bias that reflects not the hypothesised adaptation effects but rather sensitivity to the hypotheses favoured by the experimenters. This will be addressed in condition 4 (section 2.5).

\subsection{Condition 4-written nationality}

Since the mode of presentation in condition 3 was different from that in conditions 1 and 2 (image to name), we ran an additional condition, whereby the written nationality of the faces' identity was used as the adaptor. Nationality is linked to identity, but is linked to many identities. As such, it is not a finely tuned piece of semantic information. It is unlikely to affect adaptation since low-level adaptation effects are usually quite finely tuned (cf Webster and Miyahara 1997). This condition controls for mode of presentation. It is unlikely that adapting to the nationality of one of the two morph identities would suffice to significantly adapt the process tuned to that identity. This leads to the prediction that adaptation to the word 'British' should not significantly bias observers to identify the ambiguous morph as Harrison Ford. On the other hand, suppose that observers' judgments reflect a demand bias in favour of what they take to be the experimenter's hypothesis that judgments of identity shift away from the adapted identity; in this case, since they know Pierce Brosnan is British, they should be biased to say the ambiguous morph is Harrison Ford.

2.5.1 Method. We used an identical method to that in condition 3, except the adaptation stimuli were written nationalities (American or British). Since few participants were aware that Pierce Brosnan is Irish, it was felt that the nationality 'British' would suffice as the opposite of American in this experiment.

2.5.2 Results and discussion. Responses for each identity were exactly at chance levels: $50 \%$ (10 out of 20 ) responding with the identity associated to the adaptation nationality. This is, therefore, significantly different from the three conditions described above.

\subsection{Condition 5-brief presentation}

Condition 4 indicated that the mode of presentation does not influence demand characteristics. However, there is still a possibility that negative priming (Wiggs and Martin 1998) might be occurring, whereby the prolonged stimulation of one identity actually primes the alternative identity. Negative priming requires a presentation time of less than $1000 \mathrm{~ms}$ to occur. To control for this, in this condition the adaptor was presented for $250 \mathrm{~ms}$ rather than for $60 \mathrm{~s}$.

2.6.1 Method. A similar method to the general method was employed, except the adaptor was on screen for $250 \mathrm{~ms}$, which is long enough for it to be registered by the participants, but is not prolonged enough to cause adaptation (Wiggs and Martin 1998). Immediately following the adaptor, the test stimulus appeared with no mask between the two stimuli. The adaptor was the same image as that used to construct the morph.

2.6.2 Results and discussion. 30\% of responses were the non-adaptor identity (6 out of 20). This trend was in the opposite direction to the adaptation effects, but was not significantly different from chance $\left(\chi_{1}^{2}=1.667, p>0.19\right)$. This finding indicates the possibility 
of priming occurring when the stimuli are presented briefly - a finding that is similar to the effect of repetition priming observed by Ellis et al (1987). However, the effect was not significant in the present study. This may indicate the presence of demand characteristics whereby participants believe they are supposed to respond with the opposite identity. Alternatively, this methodology may not be appropriate for bringing about strong priming effects.

\subsection{Overview and discussion}

Five experimental conditions demonstrated that participants can be adapted to a specific identity from the face and the name. The results in terms of number of responses of the non-adapted identity are shown in figure 2. There were no significant differences across the same face, different face, and name adaptation stimuli. These three conditions demonstrated significant adaptation.

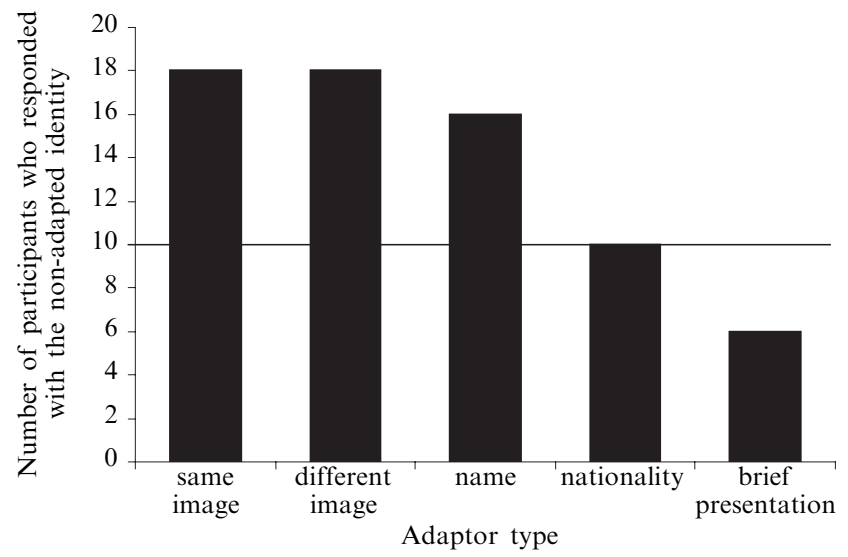

Figure 2. Number of participants who responded with the non-adapted identity split by type of adaptor for experiment 1 (pre-exposed). Chance levels are 10 participants.

There is a possibility, however, that these effects are due to a demand characteristic. In this two-alternative forced-choice paradigm, participants were pre-exposed to and aware of both of the face identities that made up the morph. It is possible that they may have felt that they were expected to give the 'opposite' answer to the adaptor identity. Thus, they could respond in a way to appear consistent with the experimenter's expectations rather than to being adapted. Because of this, we carried out a second experiment to prevent participants responding in a manner consistent with the experimenter's expectations.

\section{Experiment 2}

In experiment 2 we replicate experiment 1 using a new set of participants and controlling for the possible demand characteristic that participants were aware of the alternative identity. The only difference between experiment 2 and experiment 1 is that there was no pre-exposure phase in any condition within experiment 2. Participants were unaware of whose face the morph was constructed. As such, participants were permitted to give a completely free response to the morph. This involved asking the participants to write down who they thought the target morph looked most like on a piece of paper.

\subsection{Results}

$90 \%$ of participants chose the unadapted morph identity when the same image was used at test and adaptation (18 out of 20), akin to experiment 1, condition 1 . This was significant as before $\left(\chi_{1}^{2}=7.619, p<0.01\right) .85 \%$ of participants chose the unadapted morph identity when the images were not the same at adaptation and test (17 out of 20), 
similar to experiment 1 , condition $2\left(\chi_{1}^{2}=3.956, p<0.05\right) .75 \%$ of responses were adapted when the adaptor was the name (15 out of 20$)$, similar to experiment 1 , condition 3. This is significantly different from chance $\left(\chi_{1}^{2}=3.467, p<0.05\right)$. When the adaptor was nationality, $55 \%$ of responses were adapted (11 out of 20$)$, similar to experiment 1 , condition 4 . This was not significant $\left(\chi_{1}^{2}=0.106, p>0.75\right)$. For brief presentation (experiment 1, condition 5), 100\% of responses were the adaptor (20 out of 20), indicating total priming occurred. This was significantly different from chance, but in the opposite direction to the adaptation effects observed in all other conditions $\left(\chi_{1}^{2}=13.333, p<0.01\right)$. These results are presented in figure 3 . The results from experiments 1 and 2 were combined and subjected to a logistic regression analysis. This determined that there was no significant effect of experiment on the data $\left(\chi_{1}^{2}=1.428\right.$, $p>0.23$ ), indicating that prior knowledge of the identities that were used to construct the test morph had no significant effect on adaptation.

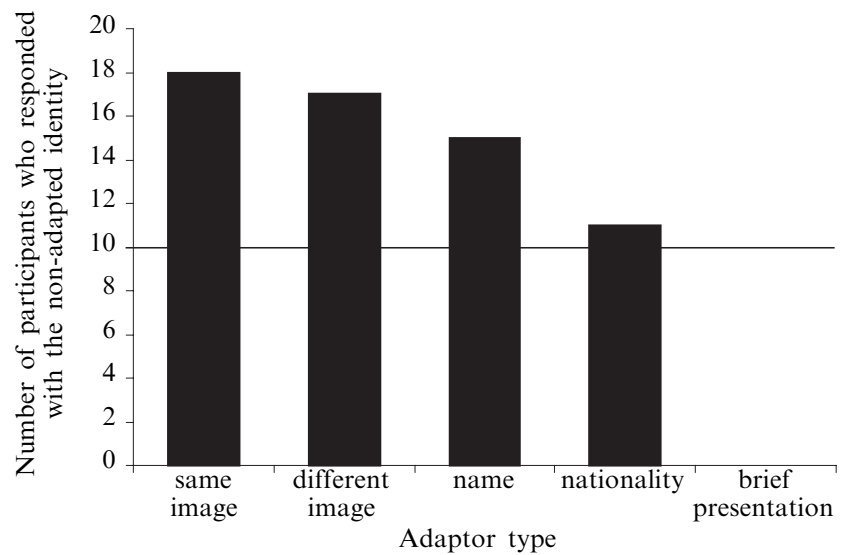

Figure 3. Number of participants who responded with the non-adapted identity split by type of adaptor for experiment 2 (free response). Chance levels are 10 participants.

\subsection{Discussion}

The data from this study have demonstrated the existence of face aftereffects that are not only visually derived. FIAEs can be produced by the same image at adaptation and test, a different image, or the name. Cross-domain identity-specific adaptation appears to have occurred in this experiment. Thus, it would seem this adaptation occurs at some high-level cognitive stage. Brief presentation did not cause adaptation, rather it caused priming. This allows us to discount an explanation based on negative priming. However, there is one cautionary point: aftereffects caused by the name were weaker (though not significantly) than aftereffects caused by the images.

Adaptation changes the detection thresholds of perceived images (Webster and MacLin 1999) and here adaptation was due to name stimuli. This is an important finding, for it suggests the existence of neuronal populations which selectively respond to particular identities. However, there is one important question that needs to be considered: what are the participants doing during the name adaptation? It is likely that presenting a name will cause the participant to think about the identity. Indeed, he or she may imagine a visual image of the person. This may mean that the route of this non-visually derived FIAE is due to imagination. Indeed, O'Craven and Kanwisher (2000) have shown that, with fMRI techniques, there is selective activation in the fusiform gyrus for perceiving and thinking about faces. Furthermore, Kosslyn (2005) has noted how imagination can affect even low-level adaptation responses such as contrast and spatial-frequency adaptation. 
Though the difference between the same image and name stimulus as the adaptor was not significant, there was a trend to suggest that fewer people could be adapted to a name stimulus. Indeed, not all participants gave a response indicative of adaptation. This indicates that there are some individual differences in the FIAE. One plausible participant variable that may influence the magnitude of the FIAE is visualisation ability. In experiment 3 we look at participants' ability to mentally visualise, and the magnitude of their FIAE.

\section{Experiment 3}

In experiments 1 and 2 we demonstrated that the FIAE can be caused by a name as well as faces. There was some indication of individual differences in this effect that might be due to the individual differences in visualisation abilities. The visual imagery questionnaire (VIQ-Marks 1973) is a useful tool for measuring participants' ability to mentally visualise (McKelvie 1994). This questionnaire measures the ability of individuals to visualise four specific scenes: a sunrise, a person, a country scene, and a shop. If visualisation abilities affect the magnitude of the FIAE, the participants who scored higher on the VIQ should demonstrate stronger FIAEs than participants who scored lower on the VIQ. To assess the possible mechanism of visualisation in the adaptation caused by names, an additional adaptation condition was incorporated, whereby participants were explicitly told to think about the person.

A multi-response psychometric procedure was employed for experiment 3. This procedure allows for an assessment of individual differences in the magnitude of the FIAE. It also allows for an assessment of the magnitude of the FIAE for different adaptor types (eg names versus images). It is also important to replicate the crossmodal FIAE with a second methodology and different face identities. This will allow for greater confidence in the results and increase generalisability of the results.

\subsection{Method}

4.1.1 Participants. Sixteen Cardiff University students undertook this experiment as partial fulfilment of a course requirement. All participants had normal or corrected vision.

4.1.2 Materials. An image of George Bush and an image of Tony Blair were collected. They were matched for dimensions $(100 \mathrm{~mm} \times 160 \mathrm{~mm})$, resolution $\left(72\right.$ pixels inch $\left.{ }^{-1}\right)$, pose, and lighting (lit from the front). A series of morphs were created with Smartmorph ${ }^{(i x}$ software, with 200 anchor points. 50 morphs were created that ranged from $100 \%$ George Bush to $100 \%$ Tony Blair in increments of $2 \%$. The $100 \%$ images were also used as the adaptors in the same image at adaptation as used to construct the test morph conditions (hereafter the same image condition). A second image of George Bush and a second image of Tony Blair were collected and used as the adaptors in the different image at adaptation from that used to construct the test morph conditions (hereafter the different image condition). These were in a different pose $\left(30^{\circ}\right)$ and under different lighting conditions (lit from the lower left) from images used to make the morph continuum. Names were displayed on screen in Palatino font, size 20, black on white. All stimuli were presented with SuperlabPro $2^{\circledR}$ research software on an RM PC.

4.1.3 Design. The four adaptors (same image as that used to construct the test morphs; different image from the one used to construct the test morphs; name; and imagined) were varied between subjects. Scores on the VIQ were split by the median score of 12 , creating a second independent variable-ability to visualise. This created a $4 \times 2$ between-subjects experiment. Participants were randomly selected to be in one of the adaptation conditions in such a way as to maintain an equal number of participants 
in all conditions. Participants were either adapted to George Bush or to Tony Blair. Since there was no effect of identity, all data are collapsed across this variable for the analysis.

4.1.4 Procedure. Participants sat at a distance of $50 \mathrm{~cm}$ from the computer screen in a darkened laboratory. They were first introduced to pictures of George Bush and Tony Blair. All participants were familiar with these identities. The experiment had three consecutive phases: baseline, adaptation, and test. The baseline phase involved presenting all the morphs (50) 10 times in a random order (thus 500 trials) to the participants. They had to decide whether each morph looked more like George Bush (by pressing the G key) or Tony Blair (by pressing the $\mathrm{T}$ key) based on the methodologies in Levitt (1971). Each morph was on screen until the participant responded (usually no longer than $900 \mathrm{~ms}$ ). Between each morph a $100 \mathrm{~ms}$ white-noise mask was on screen. The procedure for this is shown in figure 4.

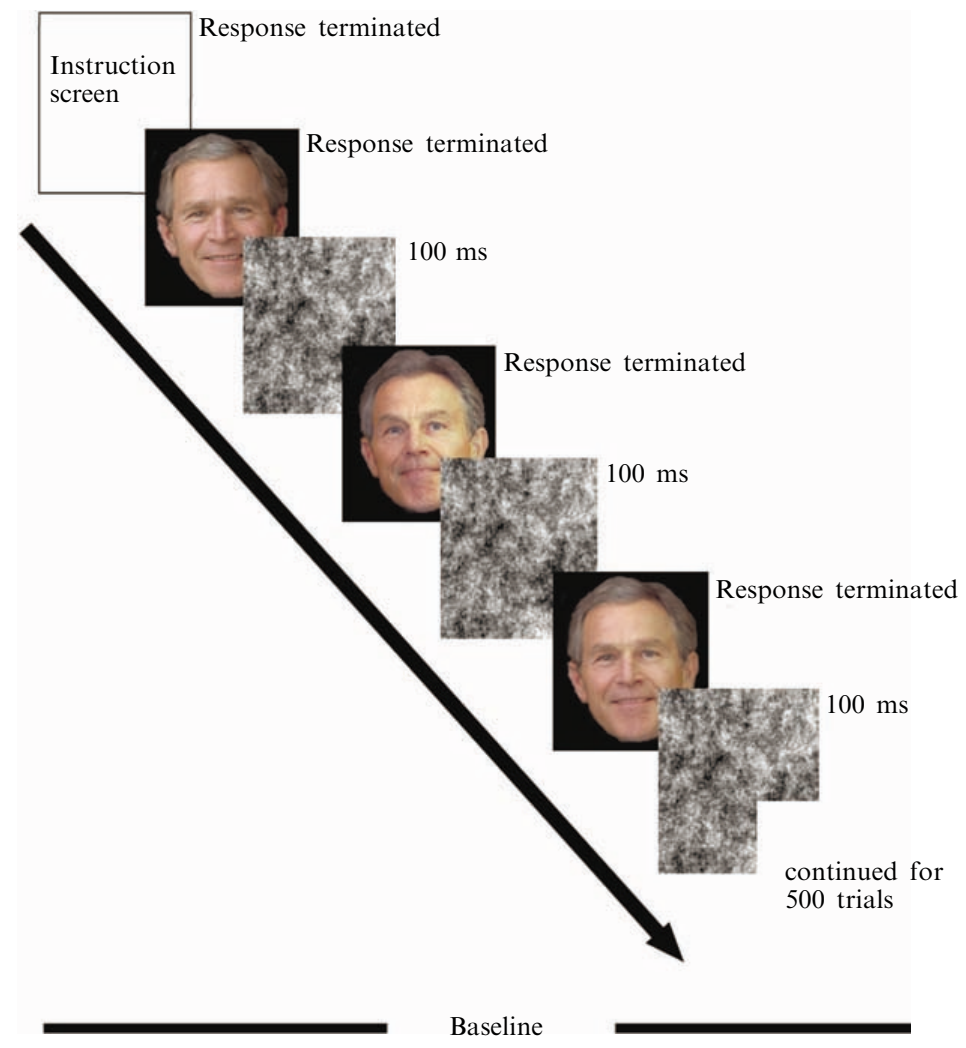

Figure 4. [In colour online.] The procedure for the baseline phase of experiment 3.

Once the baseline had finished, the participants were instructed to rest for $2 \mathrm{~min}$. Following this, participants were given the VIQ. As instructed by Marks (1973), the VIQ was read out by the experimenter, who then marked down how elaborate the visual memory was that the participants described. Prior to the experimental research, two experimenters assessed visual imagery separately with the VIQ and were found to have an inter-rater reliability of $r=0.82$.

Once the questionnaire had been completed, participants were presented with the adaptor. They were told to examine the image that was presented on screen, which was either an image of George Bush or Tony Blair, or one of their names. In the imagined adaptation conditions, participants were verbally told to think about the person whenever 
the screen was blank for the remaining duration of the experiment. The adaptor remained on screen for $60 \mathrm{~s}$.

Immediately following the adaptor, a repeat of the baseline procedure took place (all 500 trials). However, preceding each test face, participants were presented with the adaptor for a further $5 \mathrm{~s}$ (cf Hills and Lewis 2007; Rhodes et al 2003). In the test condition, the screen went blank in this $5 \mathrm{~s}$ interval and the participants thought about the adaptor identity during this time (as they had been instructed to earlier). Once the test phase had been completed, participants were thanked and debriefed fully. The test phase procedure is presented in figure 5.

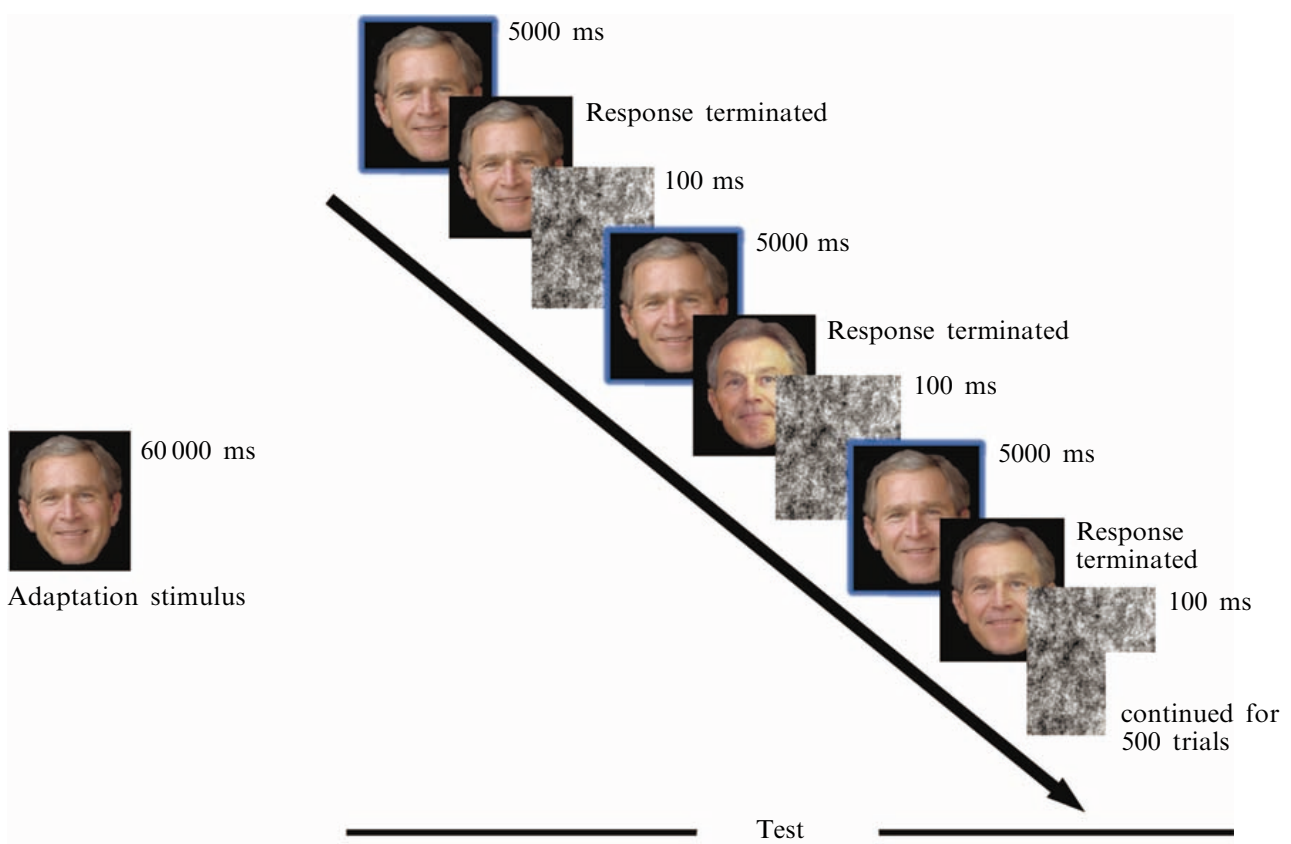

Figure 5. [In colour online.] The procedure for the test phase. Those surrounded by the box are the adaptor repeated during the test phase.

\subsection{Results}

Psychometric functions were fitted before and after adaptation for each participant with a Matlab ${ }^{\circledR}$ computer programme. These are presented in figure 6. Of interest is the location of perceptual midpoint (the PSE), the homogeneity of variance of the function, and the slope shape. For all the participants who scored highly (the upper median after a median split) on the VIQ and in the same image condition for low VIQ scorers, psychometric functions fit the observed data reliably. However, psychometric functions were not always reliable for participants who scored low on the VIQ - there was increased heterogeneity of variance (which was corrected by the computer programme) and the slope shape was shallower. Thus, psychometric functions may not be the most appropriate method for modelling the data for low visualisers.

The PSE differences preadaptation to postadaptation were tabulated and shown in table 1. The data indicate that changes in perceptual midpoint are greater for those who score high on the VIQ than for those who score low on the VIQ. These data were subjected to a $4 \times 2$ univariate between-subjects ANOVA. This revealed a significant main effect of VIQ score $\left(F_{1,8}=130.019, \mathrm{MSE}=3.603, p<0.001\right)$, whereby people who could visualise well were significantly more likely to show adaptation than those who were less good at visualisation (mean difference $=10.82, p<0.001$ ). There was also a significant main effect of adaptor $\left(F_{3,8}=62.818, \mathrm{MSE}=3.603, p<0.001\right)$. 


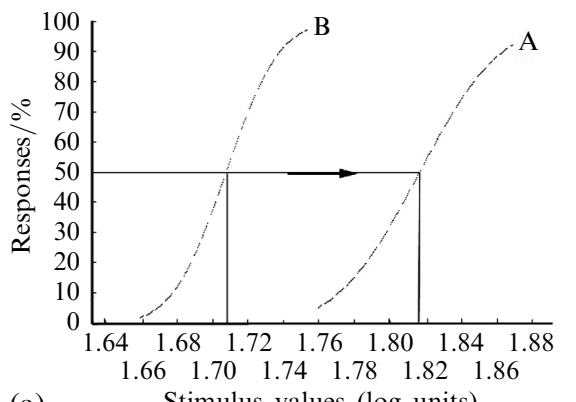

(a)
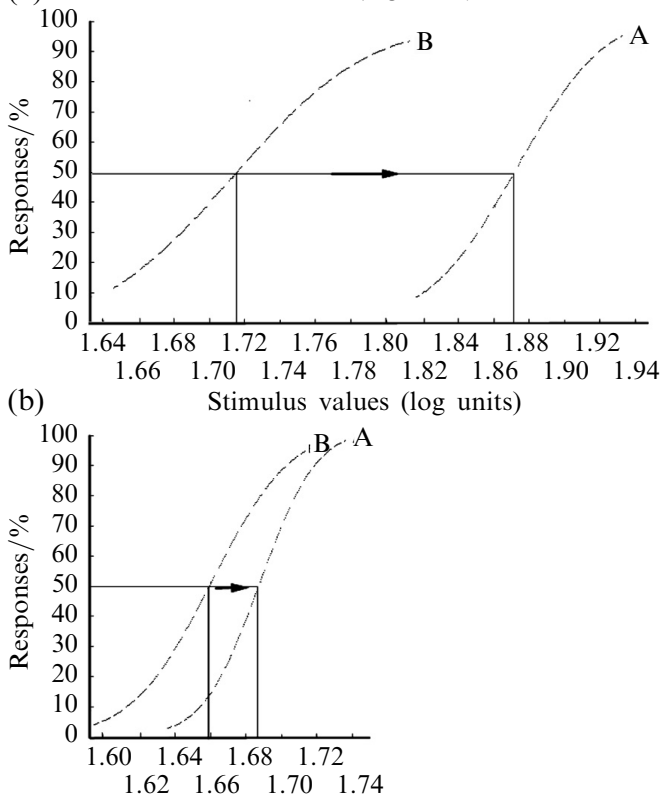

(c) Stimulus values (log units)

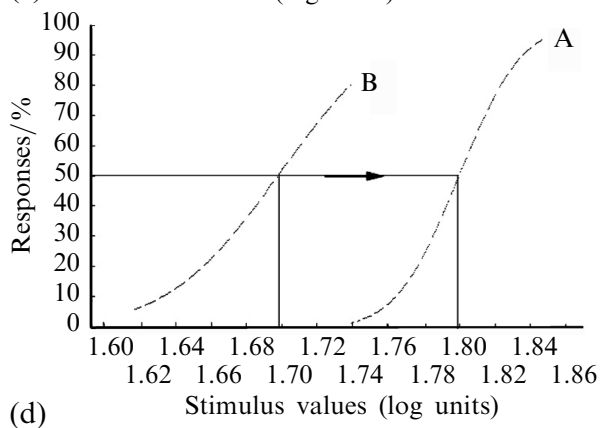

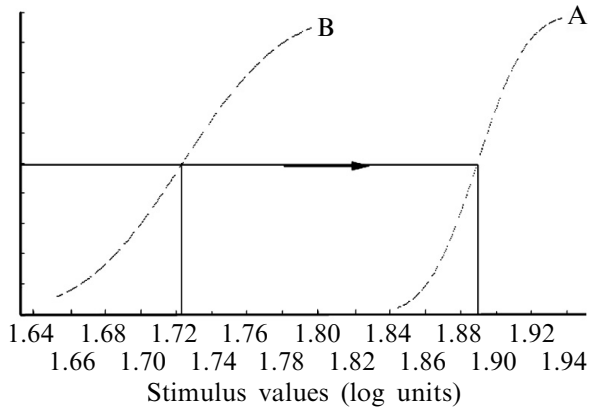

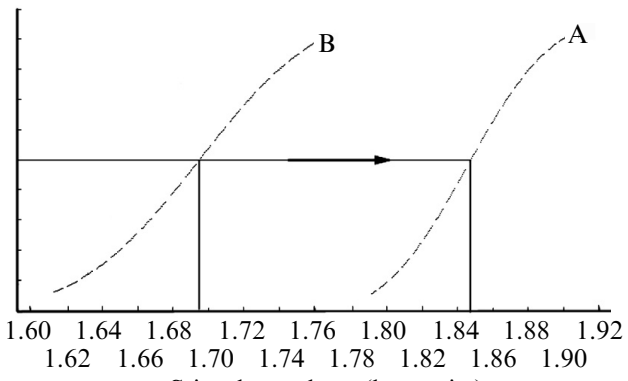

Stimulus values (log units)

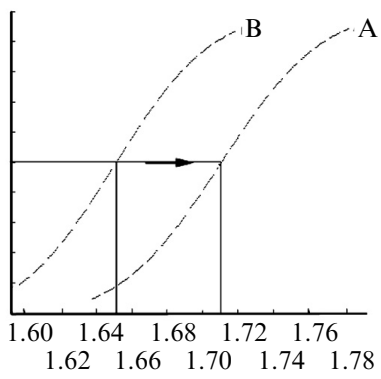

Stimulus values (log units)

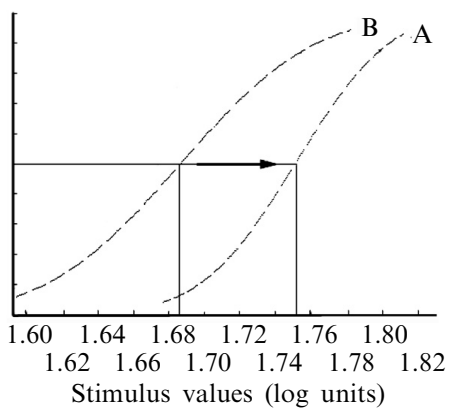

Figure 6. Psychometric functions before (B) and after (A) adaptation. Sample solution based on median PSE (point of subjective equality) for that condition. Each pair of graphs represent data from one participant. The arrow indicates significant changes in PSE point. Left panels are those adapted to George Bush. Right panels are those adapted to Tony Blair. The top four panels are participants who are scored above 12 on the visual imagery questionnaire (VIQ). (a) Same adaptation image as that used in the test morphs. (b) Different adaptation image from those used in the test morphs. (c) Name used as adaptation stimulus. (d) Imagined adaptation stimulus. Bottom four panels are participants who scored less than 12 on the VIQ. (e) Same adaptation image as that used in the test morphs. (f) Different adaptation image from those used in the test morphs. (g) Name used as adaptation stimulus. (h) Imagined adaptation stimulus. 


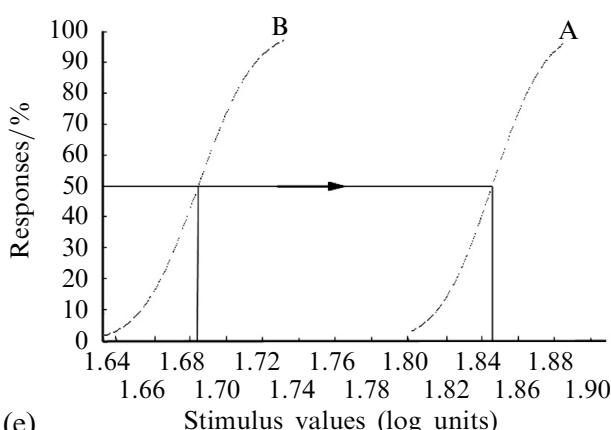

(e)

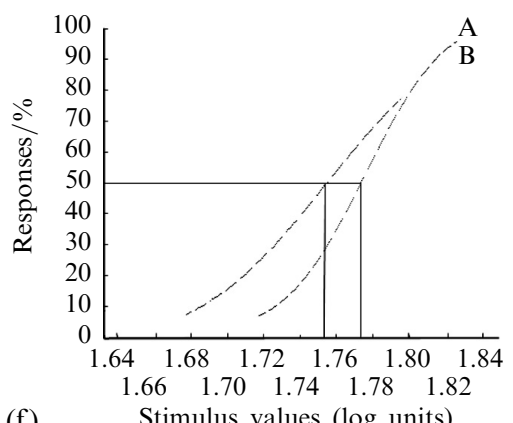

(f)

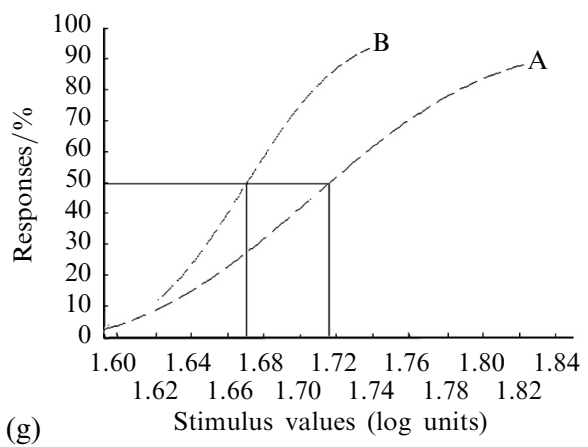

(g)

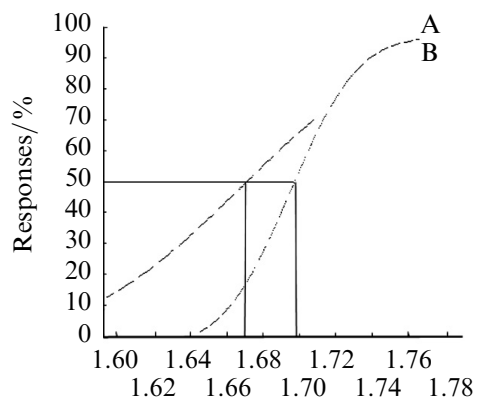

(h) Stimulus values (log units)

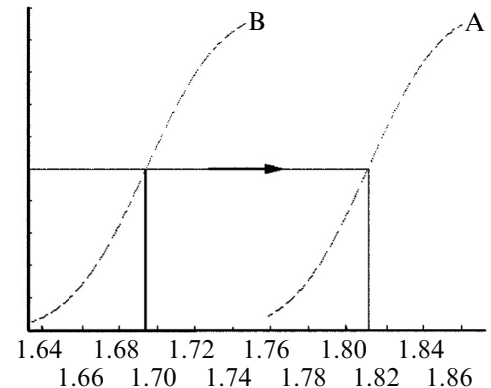

Stimulus values (log units)

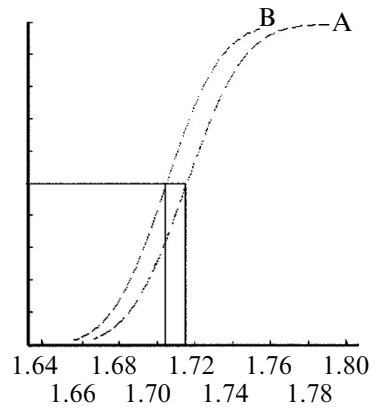

Stimulus values (log units)

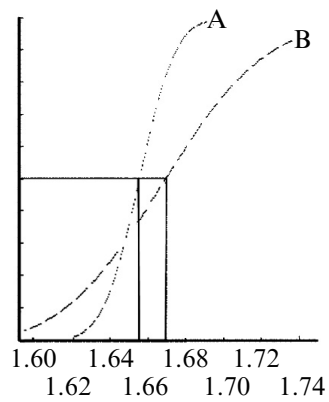

Stimulus values (log units)

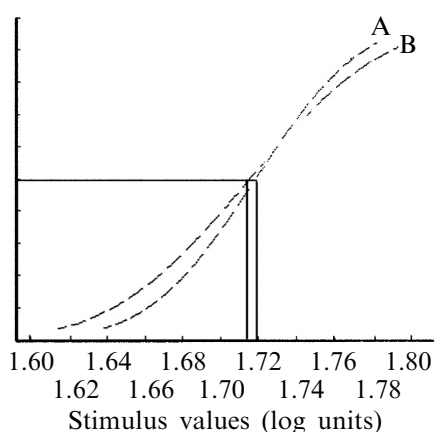

Figure 6. continued.

This was explored by means of a posteriori Tukey tests: significantly greater adaptation was observed in the same image condition over the different image condition (mean difference $=8.82, p<0.005$ ), name adaptor (mean difference $=17.16, p<0.001$ ), and imagined adaptation (mean difference $=14.14, p<0.001$ ). 
Table 1. PSE (point of subjective equality) changes preadaptation to postadaptation. Positive numbers indicate more of the adaptor identity was needed in the test morphs to see that identity. A significant shift in PSE $(p<0.05)$ is denoted by an asterix.

\begin{tabular}{lllll}
\hline $\begin{array}{llll}\text { VIQ score } \\
\text { (visualisation } \\
\text { ability) }\end{array}$ & $\begin{array}{l}\text { Adaptor } \\
\text { (compared to test } \\
\text { images) }\end{array}$ & \multicolumn{2}{l}{ Adapted to: } & Mean/\% \\
\cline { 3 - 4 } & George Bush/\% & Tony Blair/\% & \\
\hline High & same image & 23.68 & 24.54 & $24.11^{*}$ \\
& different image & 21.17 & 20.37 & $20.77^{*}$ \\
& name & 4.42 & 6.61 & $5.52^{*}$ \\
& imagined & 13.38 & 7.85 & $10.62^{*}$ \\
Low & 15.21 & 16.06 & $15.64 *$ \\
& same image & 2.43 & 0.22 & 1.33 \\
& different image & 1.71 & -1.88 & -0.09 \\
& name & 1.62 & 0.07 & 0.85 \\
\hline
\end{tabular}

These main effects were qualified by a significant interaction between VIQ score and adaptor $\left(F_{3,8}=10.013, \mathrm{MSE}=3.603, p<0.005\right)$. The interaction revealed itself in that for low-visualisation-ability participants, the FIAE was greater in the same-image condition than in the different-image condition (mean difference $=14.31, p<0.001$ ); the name adaptor (mean difference $=15.73, p<0.001$ ); and the imagined adaptation (mean difference $=14.79, p<0.001$ ). However, the differences between different-image condition, name adaptor, and imagined adaptation were not significant (all $p \mathrm{~s}>0.6$ ). For those with high-visualisation abilities, there were significant mean differences between the same-image condition and different-image condition (mean difference $=3.34$, $p<0.05$ ); the same-image condition and name adaptor (mean difference $=18.59$, $p<0.01$ ); the same-image condition and imagined adaptation (mean difference $=13.49$, $p<0.01$ ); the different-image condition and name adaptor (mean difference $=15.25$, $p<0.01$ ); the different-image condition and imagined adaptation (mean difference $=10.15$, $p<0.05$ ); and the name adaptor and imagined adaptation (mean difference $=5.10$, $p<0.05)$.

\subsection{Discussion}

Significant FIAEs were observed for participants who scored higher on the VIQ in all conditions. Participants who had lower visualisation scores demonstrated adaptation effects only when the same image was used as the adaptor and at test. These results indicate the importance of the participant's ability to mentally visualise in moderating the FIAE. In addition, the strength of the aftereffects is lower for the participants less able to visualise when the same image is used as the adaptor and in the test morphs. This is important since it suggests that even the image-based adaptation is partially mediated by the participants' ability to visualise a face. The mechanism for this mediation can only be theorised at this point and it may be that those who are better able to visualise have stronger perceptual memories or stronger links between memory and perception. Further exploration is required to understand this mediation.

A second aspect of the present data is that the magnitude of the aftereffect due to adaptation to a name is less than that when participants are told to think about the individual. One possible explanation for this is that when participants are instructed to think about someone they picture the person in their mind. Simply presenting a name may not directly link to visualisation, at least for some people, but rather lead to recalling of semantic information. Research does indicate that semantic information is more easily retrieved from names than from faces (Burton and Bruce 1993). When participants are asked to think about someone, they will usually picture him or her rather than think about semantic information (cf Stillman and Kemp 1993). 
The results from this experiment are inconsistent with those of Moradi et al (2005), who found that FIAEs could not be brought about with mental visualisation. However, in their work Moradi et al used faces that were unfamiliar to the participants and thus less easy to visualise. Moreover, the accuracy with which Moradi et al's participants visualised and their ability to visualise was not tested with any scientific accuracy as discussed in the introduction. This is especially important given the wideranging individual differences in mental imagery (eg Hatakeyama 1997; McKelvie and Demers 1979; Marks 1973).

\section{General discussion}

Taken together, these three experiments considerably add to our understanding of the FIAE. In experiment 1 we presented a series of conditions whereby aftereffects were observed when the test morph was in the same pose or a different pose from the adaptor image and when the adaptor was a name. This was irrespective of whether or not the participants knew the identities used to construct the test morph prior to adaptation, as shown in experiment 2. In experiment 3 we demonstrated that the magnitude of the FIAE was moderated by participants' ability to mentally visualise the stimuli and that the FIAE is partially mediated by mental imagery. Given that participants less able to visualise show a perceived shift in identity threshold of $15 \%$ when the same image is used as the adaptor and at test, a certain proportion of the FIAE may be explained by visual characteristics. Nevertheless, there is a significant amount of adaptation due to visualisation, given that participants who are better able to visualise show 9\% more shift in identity threshold under the same conditions. It may be that adaptation due to visualisation may add to adaptation due to visual characteristics or may be supplementary to it. In other words, there may be two routes to adaptation, one direct and one through visualisation and these may interact or add together. Further research may disentangle these possibilities.

One final issue to address is the fact that the present study involved the use of two unique identities. This is a deviation from the more established methodology of using artificially created face-anti-face pairs. The mechanism for the FIAE involving faceanti-face pairs probably involves a prototype, whereby adaptation to one identity temporarily shifts the prototype. When two unique identities are used, it is unlikely that the morph continuum goes through the prototype, and thus the mechanism for this type of FIAE may be different from that observed previously. Nevertheless, it is conceivable that adaptation to a unique identity also temporarily shifts the prototype face. Alternatively, it may cause a reduction in responding to the particular unique identity causing its identity region to be smaller (cf Voronoi model_Lewis and Johnston 1999). This will require greater identity strength to perceive that particular identity without any adjustments to the prototype. Further exploration is required to determine if these two mechanisms are indeed distinct.

These three experiments extend the FIAE to suggest it can be caused by visual and non-visual information. Moreover, the present study suggests a participant variable that causes individual differences in the FIAE. Simply thinking about someone is sufficient to alter the way that he or she is visually processed for those people who are better able to visually imagine scenes. Non-image-based identity adaptation is thus moderated by participants' ability to mentally visualise faces, whereas image-based identity adaptation may be mediated by participants' ability to mentally visualise faces, since participants who are better able to visualise images experience a greater FIAE. This indicates that the FIAE can be caused by multimodal adaptation and is linked to the participants' ability to mentally visualise a face. 
Acknowledgments. This research was supported by grant PTA-030-2003-00524 from the ESRC to Peter Hills. We would like to thank Thomas Busey, Peter Hancock, Alice O'Toole, and two anonymous reviewers for comments on previous drafts of this paper. The probit and heterogenous probit functions were programmed in Matlab ${ }^{(m)}$ by Rhodri Woodhouse at Cardiff University.

\section{References}

Amedi A, Malach R, Pascual-Leone A, 2005 "Negative BOLD differentiates visual imagery and perception" Neuron $5859-872$

Benton C P, Jannings S J, Chatting D J, 2006 "Viewpoint dependence in adaptation to facial identity" Vision Research 463313 - 3325

Burton A M, Bruce V, 1993 "Naming faces and naming names: Exploring an interactive activation model of person recognition" Memory $1457-480$

Burton A M, Jenkins R, Hancock P J B, White D, 2005 "Robust representations for face recognition: the power of averages" Cognitive Psychology 51 256-284

Bywaters M, Andrade A, Turpin G, 2004 "Determinants of the vividness of visual imagery: the effects of delayed recall, stimulus affect and individual differences" Memory 12 479-488

Carbon C C, Leder H, 2005 "Face adaptation: changing stable representations of familiar faces within minutes?" Advances in Experimental Psychology 1 1-7

Cui X, Jeter C B, Yang D, Montague P R, Eagleman D M, 2007 "Vividness of mental imagery: individual variability can be measured objectively" Vision Research $47474-478$

Ellis A W, Young A W, Flude B M, Hay D C, 1987 "Repetition priming of face recognition" Quarterly Journal of Experimental Psychology A $39193-210$

Favreau O E, 1979 "Persistence of simple and contingent motion aftereffects" Perception \& Psychophysics $26187-194$

Hanley C, Zerbolio D J, 1965 "Developmental changes in five illusions measured by the up-and-down method" Child Development $36437-452$

Hasnain N, Husain M G, 1980 "Is creative imagination equivalent to visual \& auditory imaginations" Asian Journal of Psychology and Education $649-52$

Hatakeyama T, 1997 "Adults and children with high imagery show more pronounced perceptual priming effect" Perceptual \& Motor Skills $941315-1329$

Hills P J, Lewis M B, 2007 "Temporal limitation of the Navon effect on face recognition" Perceptual \& Motor Skills $104501-509$

Hulbert A, 2001 "Trading faces" Nature Neuroscience 4 3-5

Ishai A, Schmidt C F, Boesiger P, 2005 "Face perception is mediated by a distributed cortical network" Brain Research Bulletin 6787 -93

Issac A R, Marks D F, 1994 "Individual differences in mental imagery experience: developmental changes and specialization" British Journal of Psychology 85 479-500

Jeffery L, Rhodes G, Busey T, 2006 "View-specific coding of face shape" Psychological Science $17501-505$

Jiang F, Blanz V, O'Toole A, 2006 "Probing the visual representation of faces with adaptation: a view from the other side of the mean" Psychological Science 17 493-500

Jiang F, Blanz V, O'Toole A, 2007 "The role of familiarity in three-dimensional view-transferability of face identity adaptation" Vision Research $47525-531$

Jones P D, Holding D H, 1975 "Extremely long-term persistence of the McCollough effect" Journal of Experimental Psychology: Human Perception and Performance 4 323-327

Johnston R A, Barry C, 2006 "Repetition priming of access to biographical information from faces" Quarterly Journal of Experimental Psychology 59326 - 339

Kanwisher N, McDermott J, Chun M M, 1997 "The fusiform face area: a module in human extrastriate cortex specialized for face perception" Journal of Neuroscience $174302-4311$

Kersten D, Troje N F, Bülthoff H H, 1996 "Phenomenal competition for poses of the human head" Perception $25367-368$

Kosslyn S M, 2005 "Mental images and the brain" Cognitive Neuropsychology $22333-347$

Kosslyn S M, Alpert N M, Thompson W L, Chabris C F, Rauch S K, Anderson A K, 1994 "Identifying objects seen from different viewpoints: a PET investigation" Brain 117 1055-1071

Kosslyn S M, Alpert N M, Thompson W L, Malikovic V, Weise S B, Chabris C F, Hamilton S E, Rauch S L, Buonanno F S, 1993 "Visual mental imagery activates topographically organized visual cortex: PET investigations" Journal of Cognitive Neuroscience 5263 -287

Kreiman G, Koch D, Fried I, 2000 "Imagery neurons in the human brain" Nature 408 357-361

Leopold D A, O'Toole A J, Vetter T, Blanz V, 2001 "Prototype-referenced shape encoding revealed by high-level after effects" Nature Neuroscience 4 89-94 
Leopold D A, Rhodes G, Müller K, Jeffery L, 2005 "The dynamics of visual adaptation to faces" Proceedings of the Royal Society of London, Series B $272897-904$

Levitt H, 1971 "Transformed up-down methods in psychoacoustics" Journal of the Acoustical Society of America $49467-477$

Lewis M B, Johnston R A, 1999 "A unified account of the effect of caricaturing faces" Visual Cognition $61-42$

McKelvie S J, 1994 "The Vividness of Visual Imagery Questionnaire as a predictor of facial recognition memory performance" British Journal of Psychology 85 93-104

McKelvie S J, Demers E G, 1979 "Individual differences in reported visual imagery and memory performance" British Journal of Psychology $7051-57$

Marks D F, 1973 "Visual imagery differences in the recall of pictures" British Journal of Psychology $6417-24$

Moradi D, Koch C, Shimojo S, 2005 "Face adaptation depends on seeing the face" Neuron 45 $169-175$

O'Craven K M, Kanwisher N, 2000 "Mental imagery of faces and places activates corresponding stimulus-specific brain regions" Journal of Cognitive Neuroscience 12 1013-1023

Pourtois G, Schwartz S, Seghier M L, Lazeyras F, Vullieumier P, 2005 "Portraits or people? Distinct representations of face identity in the human visual cortex" Journal of Cognitive Neuroscience 17 $1043-1057$

Rhodes G, Jeffery L, Watson T L, Clifford C W G, Nakayama K, 2003 "Fitting the mind to the world: Face adaptation and attractiveness after effects" Psychological Science 14 558-566

Richardson A, 2000 "Individual differences in visual imagination imagery", in Individual Differences in Conscious Experience Eds R G Kunzendorf, B Wallace (Amsterdam: John Benjamins) pp $125-146$

Richardson A, McAndrew F, 1990 "The effects of photic stimulation and private self-consciousness on the complexity of visual imagination imagery" British Journal of Psychology 81 381-394

Ryu J, Chaudhuri A, 2006 "Representations of familiar and unfamiliar faces as revealed by viewpoint-aftereffects" Vision Research 464059 - 4063

Sekuler R, Blake R, 2001 Perception (New York: McGraw-Hill)

Spillmann L, 1994 "The Hermann grid illusion: a tool for studying human perceptive field organization" Perception $23691-708$

Stillman J, Kemp T, 1993 "Visual versus auditory imagination: image qualities, perceptual qualities, and memory" Journal of Mental Imagery 17181 - 194

Troje N F, Bülthoff H H, 1996 "Face recognition under varying poses: the role of texture and shape" Vision Research 361761 - 1771

Valentine T, 1991 "A unified account of the effects of distinctiveness, inversion and race on face recognition" Quarterly Journal of Experimental Psychology A 43 161-204

Webster M A, MacLin O H, 1999 "Figural after effects in the perception of faces" Psychonomic Bulletin \& Review 6 647-653

Webster M A, Miyahara E, 1997 "Contrast adaptation and the spatial structure of natural images" Journal of the Optical Society of America A 14 2355-2366

Wiggs C L, Martin A, 1998 "Properties and mechanisms of perceptual priming" Current Opinion in Neurobiology $8227-233$

Young A W, Flude B M, Hellawell D J, Ellis A W, 1994 "The nature of semantic priming effects in the recognition of familiar people" British Journal of Psychology 85393 - 411

Zhao L, Chubb C, 2001 "The size-tuning of the face-distortion after-effect" Vision Research 41 $2979-2994$ 


\section{PERTEPTION}

VOLUME 372008

www.perceptionweb.com

Conditions of use. This article may be downloaded from the Perception website for personal research by members of subscribing organisations. Authors are entitled to distribute their own article (in printed form or by e-mail) to up to 50 people. This PDF may not be placed on any website (or other online distribution system) without permission of the publisher. 